\title{
Dispepsia en niños: ¿orgánica o funcional? Experiencia en un hospital de tercer nivel
}

\author{
Erick M. Toro-Monjaraz*, Karen Ignorosa-Arellano, Karen Raya-Aguirre, Alejandro Loredo-Mayer, \\ Roberto Cervantes-Bustamante, Flora Zárate-Mondragón, Jose Cadena-León y Jaime Ramírez-Mayans \\ Departamento de Gastroenterologia y Nutrición Pediátrica, Instituto Nacional de Pediatría, Ciudad de México, México
}

\section{Resumen}

Introducción: La dispepsia consiste en un conjunto de síntomas que pueden tener origen orgánico o funcional. El objetivo de este estudio fue describir las principales causas de la dispepsia y su evolución clínica en niños en un hospital de tercer nivel. Material y métodos: Estudio retrospectivo en niños con dispepsia. Los pacientes fueron sometidos a endoscopia con toma de biopsia y prueba de urea rápida para Helicobacter pylori. En caso de endoscopia y biopsia normal, se tomó prueba de hidrogeniones en aliento. En todos los casos se dio seguimiento para evaluar la mejoría de síntomas. Resultados: Se incluyeron 100 niños, de los cuales 52 eran niñas; la edad media fue de 8.59 años. Se encontró esofagitis y gastropatía erosiva en el $54 \%$ de los casos ( $n=54)$, infección por $H$. pylori en el $12 \%$ ( $n=12)$, sobrecrecimiento bacteriano del intestino delgado en el $12 \%$ ( $n=12)$ y dispepsia funcional en el $20 \%(n=20)$. Conclusión: En niños con dispepsia se deben de descartar primero causas orgánicas antes de diagnosticar dispepsia funcional. En términos generales consideramos que es necesario un abordaje escalonado que incluya endoscopia con toma de biopsia, búsqueda de $\mathrm{H}$. pylori y una prueba de hidrogeniones.

PALABRAS CLAVE: Dispepsia. Dolor abdominal crónico. Niños. Sobrecrecimiento bacteriano del intestino delgado.

\section{Dyspepsia in children: Organic or functional? Experience in a tertiary care hospital}

\begin{abstract}
Introduction: Dyspepsia comprises a group of symptoms that can either have an organic cause or functional origin. The purpose of this study was to describe the main causes of dyspepsia and the its clinical evolution in children cared for in a tertiary care hospital. Material and methods: Retrospective study in children with dyspepsia. Patients underwent endoscopy and rapid urease test to detect the presence of Helicobacter pylori. In case of normal endoscopy and biopsy, hydrogen breath test was performed. In all cases, follow-up was provided in order to evaluate symptom improvement. Results: One hundred children were included, out of whom 52 were girls; mean age was 8.59 years. Esophagitis or erosive gastropathy were found in $54 \%$ of the cases $(n=54)$. H. pylori infection in $12 \%(n=12)$, small intestine bacterial overgrowth in $12 \%(n=12)$, and functional dyspepsia in $20 \%(n=20)$. Conclusion: In children with dyspepsia, organic causes should first be ruled out before dyspepsia being characterized as functional. In general terms, we consider that a stepped approach that includes endoscopy with biopsy, search for $\mathrm{H}$. pylori and hydrogen breath test is necessary.
\end{abstract}

KEY WORDS: Dyspepsia. Chronic abdominal pain. Children. Small intestinal bacterial overgrowth.

Correspondencia:

*Erick M. Toro-Monjaraz

E-mail: emtoromonjaraz@ hotmail.com
Fecha de recepción: 19-01-2021

Fecha de aceptación: 27-04-2021

DOI: $10.24875 / G M M .21000050$
Gac Med Mex. 2021;157:491-496

Disponible en PubMed

www.gacetamedicademexico.com

0016-3813/๑ 2021 Academia Nacional de Medicina de México, A.C. Publicado por Permanyer. Este es un artículo open access bajo la licencia CC BY-NC-ND (http://creativecommons.org/licenses/by-nc-nd/4.0/). 


\section{Introducción}

La enfermedad ácido-péptica o la dispepsia es un síndrome clínico relacionado con la región gastroduodenal del tracto gastrointestinal superior, los síntomas incluyen la presencia o persistencia de dolor recurrente o malestar en el epigastrio, saciedad temprana, dolor posprandial, náuseas, pirosis, eructos o hipo excesivo, anorexia, pérdida de peso, acidez, dolor abdominal periumbilical y regurgitación $n^{1,2}$.

El impacto económico de atención de un paciente con esta patología va de 699 a 724 USD de forma anual, con afectación de la calidad de vida de los pacientes y de la familia ${ }^{3-5}$.

La dispepsia puede ser la manifestación de enfermedades orgánicas, sistémicas o metabólicas, o no tener una causa aparente, como en la dispepsia funcional. De acuerdo con los criterios de Roma IV, la dispepsia funcional se define como la presencia de al menos cuatro días por mes en los últimos dos meses de uno o más de los siguientes síntomas: plenitud posprandial, saciedad temprana, dolor o ardor en epigastrio no asociado con la defecación. La evaluación exhaustiva descarta otros problemas médicos ${ }^{6}$.

La importancia de conocer las causas principales de la dispepsia radica en establecer un diagnóstico y tratamiento adecuado.

Dentro de las principales causas descritas en la población pediátrica se encuentran: la enfermedad ulcerosa péptica primaria; agentes infecciosos como Helicobacter pylori y otras menos frecuentes como citomegalovirus, virus herpes simple, virus de la influenza A, Treponema pallidum, Candida albicans, histoplasmosis; estados de hipersecreción como síndrome de Zollinger-Ellison, medicamentos como analgésicos no esteroideos, esteroides, alcohol y ácido valproico, entre otras ${ }^{7}$.

En México se han realizado estudios para conocer cuáles son las causas de dolor abdominal crónico en niños, encontrando causas orgánicas como las principales razones del dolor, sin embargo, en estos estudios no se hizo diferencia entre la sintomatología de los pacientes ni seguimiento para evidenciar la resolución de los síntomas ${ }^{8,9}$. Los objetivos de este estudio fueron identificar y describir las principales causas de dispepsia en niños y adolescentes mexicanos atendidos en un hospital de referencia pediátrica en la Ciudad de México y comparar la mejoría antes y después del tratamiento establecido.

\section{Material y métodos}

Se realizó un estudio retrospectivo, observacional y analítico en niños con dispepsia.

La dispepsia se definió por uno o más de los siguientes síntomas: dolor en epigastrio, sensación de saciedad temprana, plenitud, eructos, sensación de ardor, náuseas y vómitos continuos o intermitentes al menos cuatro días al mes durante al menos dos meses previos.

Se realizó una historia clínica y exploración física a todos los niños y se solicitó estudio coproparasitoscópico en serie de tres, en caso de cumplir con criterios de dispepsia y no tener evidencia de parasitosis en los coproparasitoscópicos, se realizó estudio de endoscopia de tracto gastrointestinal superior con toma de biopsias de duodeno, estómago y esófago previo consentimiento $y / 0$ asentimiento informado. Durante el estudio endoscópico a todos los pacientes se les realizó prueba de urea rápida para detección de $H$. pylori.

\section{Diagnóstico de sobrecrecimiento bacteriano del intestino delgado}

En caso de no presentar datos endoscópicos que justificaran la sintomatología se les realizó prueba de hidrogeniones en aliento con equipo Gastrolyzer ${ }^{\circledR}$, con las siguientes especificaciones: suspensión de inhibidores de bomba de protones o cualquier antiácido al menos dos semanas previo al estudio. Este estudio se realizó en ayuno y se le administró al paciente $0.5 \mathrm{~g} / \mathrm{kg}$ de lactulosa, máximo $10 \mathrm{~g}$ y se realizaron mediciones cada 20 minutos a lo largo de 3 horas. Se consideró positivo el estudio para sobrecrecimiento bacteriano del intestino delgado (SBID) cuando se evidenció incremento de más de 20 ppm respecto a la basal antes del minuto 90 .

\section{Diagnóstico de Helicobacter pylori}

Las biopsias gástricas se tomaron de acuerdo con el protocolo de Sídney y se evaluaron mediante una prueba rápida de ureasa y un examen histológico. La infección por $H$. pylori se definió mediante dos pruebas invasivas positivas, esto de acuerdo con las últimas guías publicadas en niños ${ }^{10}$.

Todos los pacientes con evidencia grave de inflamación en el tracto gastrointestinal superior (esofagitis, duodenitis, gastritis y úlcera) recibieron inhibidor de la bomba de protones durante cuatro semanas y en caso 
necesario se agregó sucralfato $80 \mathrm{mg} / \mathrm{kg} / \mathrm{día}$ y se realizó endoscopia de control al término del tratamiento.

Los pacientes con SBID fueron tratados con rifaximina por siete días y probióticos (Saccharomyces boulardii o Lactobacillus GG) por dos semanas. Al grupo de pacientes positivos para SBID se les realizó test de hidrogeniones de control seis semanas después.

Los pacientes con resultado positivo para infección por $H$. pylori recibieron tratamiento estándar (amoxicilina $50 \mathrm{mg} / \mathrm{kg} / \mathrm{día}$, claritromicina $15 \mathrm{mg} / \mathrm{kg} /$ día e inhibidor de la bomba de protones diariamente por 15 días). Para valorar la erradicación de $H$. pylori además de la valoración clínica se les realizó prueba de aliento con urea marcada con carbono 13.

Consideramos la dispepsia funcional según los criterios de Roma IV en todo niño con sintomatología previamente descrita y con endoscopia normal, prueba de test de hidrogeniones negativa y sin infección por $H$. pylori. El tratamiento de esta entidad incluyó inhibidores de bomba de protones, procinéticos y valoración por salud mental.

\section{Resultados}

De los 100 sujetos, 52 eran niñas y 48 niños, la edad media fue de 8.59 años, 49 niños estaban eutróficos de acuerdo con el índice de masa corporal. Las características demográficas de los pacientes se muestran en la tabla 1. En cuanto a los diagnósticos establecidos, la esofagitis y la gastropatía erosiva en conjunto correspondió al 54\% $(n=54)$ de los casos (Tabla 2), seguido de la dispepsia funcional en el $20 \%$ de los casos y sobrecrecimiento bacteriano e infección por $H$. pylori representaron el $12 \%$ cada una, solo encontramos úlcera péptica en dos casos, no asociada a H. pylori.

En cuanto a la sintomatología, la saciedad temprana fue el síntoma predominante $(75 \%)$, seguido de anorexia (65\%) y dolor en epigastrio (59\%), sin embargo, de acuerdo con el diagnóstico etiológico la sintomatología fue diferente, los niños diagnosticados con esofagitis tuvieron como síntoma predominante la anorexia (71\%) y el dolor en epigastrio (66\%), en cuanto a la dispepsia funcional los síntomas que predominaron fueron la saciedad temprana, distensión abdominal y dolor en epigastrio; en los niños con $\mathrm{H}$. pylori fue más frecuente la saciedad temprana y el dolor en epigastrio, y finalmente, en los niños con SBID predominó el dolor periumbilical difuso, la saciedad temprana y la anorexia (Tabla 3).
Tabla 1. Características generales de los participantes. *Sobrecrecimiento bacteriano del intestino delgado

\begin{tabular}{|l|c|}
\hline Variable & $\mathrm{n}=100 \%$ \\
\hline $\begin{array}{l}\text { Sexo } \\
\text { Mujer } \\
\text { Hombre }\end{array}$ & 48 \\
\hline Edad & 58 \\
(media) & $8.59(2-14$ años $)$ \\
\hline IMC & \\
\hline Estado nutricional & 18.49 \\
\hline Eutrófico & \\
Desnutrición & 49 \\
Sobrepeso & 14 \\
Obesidad & 17 \\
\hline
\end{tabular}

IMC: índice de masa corporal.

Tabla 2. Diagnóstico de los pacientes con dispepsia

\begin{tabular}{l|c} 
Diagnóstico & $\mathrm{n}=100 \%$ \\
\hline Esofagitis erosiva & 45 \\
Dispepsia funcional & 20 \\
SBID & 12 \\
Helicobacter pylori & 12 \\
Gastropatía erosiva & 9 \\
Úlcera & 2
\end{tabular}

SBID: sobrecrecimiento bacteriano del intestino delgado.

En cuanto a la sintomatología, hubo una mejoría del $76 \%$ en toda la población con el tratamiento específico para cada patología.

Los pacientes con SBID tuvieron una mejoría del $91 \%(p=0.029)$, los de esofagitis erosiva tuvieron mejoría en el $75.5 \%$ ( $p=0.008)$, en dispepsia funcional mejoría del $80 \%(p=0.018)$ y finalmente los pacientes con infección por $H$. pylori tuvieron una mejoría del 66.6\% ( $p=0.029)$ (Tabla 4).

\section{Discusión}

La dispepsia es un conjunto de síntomas que pueden representar diferentes enfermedades. Estudios previos han evaluado la etiología de estos síntomas, Hyams, et al. realizaron un estudio en 127 niños, de los cuales solo a 56 se les realizó endoscopia de acuerdo con el criterio del médico tratante, encontrando una prevalencia de dispepsia funcional en el $62 \%$ de este subgrupo y el resto presentaba algún hallazgo endoscópico que 
Tabla 3. Descripción de la sintomatología de acuerdo con el diagnóstico

\begin{tabular}{|c|c|c|c|c|c|c|c|}
\hline Sintomas & $\begin{array}{c}\text { Esofagitis erosiva } \\
45\end{array}$ & $\begin{array}{c}\text { Dispepsia } \\
\text { funcional } \\
20\end{array}$ & $\begin{array}{c}\text { SBID } \\
12\end{array}$ & $\begin{array}{c}\text { Helicobacter pylori } \\
12\end{array}$ & $\begin{array}{c}\text { Gastropatía } \\
\text { erosiva } \\
9\end{array}$ & $\begin{array}{c}\text { Úlcera } \\
2\end{array}$ & $\begin{array}{c}\text { Total } \\
100\end{array}$ \\
\hline Epigastralgia & $30(66.6 \%)$ & $16(85 \%)$ & $2(16.6 \%)$ & $8(66.6 \%)$ & $2(22.2 \%)$ & $1(50 \%)$ & 59 \\
\hline $\begin{array}{l}\text { Dolor abdominal } \\
\text { periumbilical }\end{array}$ & $15(33.3 \%)$ & $4(25 \%)$ & $10(83 \%)$ & $4(33.3 \%)$ & $7(77.7 \%)$ & $1(50 \%)$ & 41 \\
\hline Náuseas & $13(28.8 \%)$ & $6(30 \%)$ & $4(33.3 \%)$ & $6(50 \%)$ & $3(33.3 \%)$ & $1(50 \%)$ & 33 \\
\hline Vómitos & $11(24.4 \%)$ & $5(25 \%)$ & $3(25 \%)$ & $3(25 \%)$ & $2(22.2 \%)$ & 0 & 24 \\
\hline Saciedad temprana & $29(64.4 \%)$ & $18(90 \%)$ & $10(83 \%)$ & $9(75 \%)$ & $7(77.7 \%)$ & $2(100 \%)$ & 75 \\
\hline Eructos & $14(31.1 \%)$ & $15(75 \%)$ & $5(41.6 \%)$ & $4(33 . \%)$ & $3(33.3 \%)$ & $1(50 \%)$ & 42 \\
\hline Anorexia & $32(71.1 \%)$ & $13(65 \%)$ & $11(91.6 \%)$ & $2(16.6 \%)$ & $6(66.6 \%)$ & $1(50 \%)$ & 5 \\
\hline Distensión abdominal & $20(44.4 \%)$ & $18(90 \%)$ & $9(75 \%)$ & $5(41.6 \%)$ & $2(22.2 \%)$ & 0 & 54 \\
\hline
\end{tabular}

SBID: sobrecrecimiento bacteriano del intestino delgado.

Tabla 4. Mejoría de la sintomatología con tratamiento específico

\begin{tabular}{l|c|c|}
\hline Diagnostico & Mejoría & $p$ \\
\hline Esofagitis erosiva & $34(75.5 \%)$ & 0.008 \\
\hline Dispepsia funcional & $16(80 \%)$ & 0.018 \\
SBID & $11(91 \%)$ & 0.029 \\
Helicobacter pylori & $8(66.6 \%)$ & 0.029 \\
Gastropatía erosiva & $6(55.5 \%)$ & 0.06 \\
\hline Úlcera & $2(100 \%)$ & 0.09
\end{tabular}

SBID: sobrecrecimiento bacteriano del intestino delgado.

justificaba el dolor ${ }^{11}$, en nuestro estudio encontramos hallazgos endoscópicos (gastropatía erosiva, esofagitis) que justificaban la sintomatología en el $54 \%$ de los niños, aunque menor a lo reportado por Adeniyi, et al. en Lagos (Nigeria), en donde encontraron hallazgos endoscópicos en el $87 \%$ de los niños y adolescentes que acudieron con datos de dispepsia ${ }^{12}$. En nuestros pacientes posterior al manejo establecido hubo una mejoría de los síntomas en el $75 \%$ de los niños que presentaban esofagitis y solo en el $50 \%$ de los que se presentaron con gastropatía erosiva.

H. pylori es una causa de síntomas dispépsicos, sin embargo su frecuencia en niños y la causalidad con el dolor abdominal es un tema de discusión, en una revisión sistemática de 38 artículos no se encontró asociación entre la sintomatología gastrointestinal en niños y $\mathrm{H}$. pylori en pacientes que acuden a primer nivel de atención, sin embargo, sí existió asociación con dolor epigástrico e infección por $H$. pylori en seis estudios realizados intrahospitalariamente con una odds ratio agrupada de 2.87 (intervalo de confianza del 95\%: 1.62-5.09) ${ }^{13}$.

En nuestro estudio $H$. pylori fue diagnosticado con dos pruebas (prueba rápida de urea y biopsia) en el $12 \%$ de los pacientes, la saciedad temprana y el dolor en epigastrio fueron los síntomas más frecuentes en este grupo de pacientes (75 y $66 \%$ respectivamente) presentado mejoría posterior a la erradicación el $66 \%$ de los niños; esto puede explicarse por resistencias a los antibióticos utilizados o incluso a que esta bacteria no sea la responsable de la sintomatología. Otros estudios han evaluado el papel que juega $H$. pylori en el desarrollo de síntomas dispépsicos. Farrell, et al. encontraron mejoría de los síntomas en niños a los cuales se les erradicó $H$. pylori y en su mayoría mejoría a largo plazo ${ }^{14}$. Otro estudio realizado en Turquía también mostró la mejoría de síntomas dispépsicos en el grupo de pacientes a los cuales se les erradico H. pylori ${ }^{15}$. Consideramos que dada la evidencia demostrada, en caso de diagnosticarse $H$. pylori por métodos invasivos es necesario dar tratamiento de erradicación, aunque probablemente no sea la principal causa de dispepsia en niños.

EI SBID se ha descrito como una causa de síndrome de intestino irritable en adultos y recientemente se ha descrito como causa de dispepsia ${ }^{16,17}$, sin embargo en niños existen pocos estudios que han asociado el SBID con dolor abdominal crónico ${ }^{18}$. Hasta donde sabemos este es el primer estudio realizado en niños en donde se ha asociado la dispepsia 
con SBID. En un estudio reciente en pacientes con trastornos funcionales gastrointestinales, controles sanos y enfermedad inflamatoria intestinal, utilizando un dispositivo para biopsia con técnica aséptica y cuantificando mediante reacción en cadena de la polimerasa cuantitativa la carga de bacterias, encontraron un número elevado de bacterias en duodeno en pacientes con Trastronos funcionales gastrointestinales (TGF) comparado con sujetos sanos y solo el $15 \%$ resultó positiva en el test de hidrogeniones con glucosa y el $13.3 \%$ con metano, lo que demuestra que el test de hidrogeniones no necesariamente refleja lo que sucede con el número de bacterias en duodeno en el SBID ${ }^{19}$. En nuestro estudio encontramos que el $12 \%$ (12 niños) de los niños tuvo SBID y posterior al tratamiento mejoró el $90 \%$ de estos (11 niños), por lo que se abre una nueva posibilidad en la etiología de la dispepsia en niños y necesariamente su investigación y comprobación.

En cuanto a la explicación de por qué el SBID causa dispepsia, Tziatzios, et al. lo resumen como una cascada de eventos que resulta en inflamación transmural, ganglionitis mientérica, dismotilidad e hiperalgesia visceral secundaria a la presencia excesiva de bacterias en el intestino delgado ${ }^{20}$. Este es un estudio que permite tener un acercamiento sobre las principales causas de dispepsia en un hospital de tercer nivel, lo cual puede diferir con lo que se puede observar en un primer nivel de atención, sin embargo, permite establecer las prioridades en los métodos diagnósticos y el tratamiento empleado para evitar procedimientos innecesarios. Las debilidades de este estudio son que no se utilizó un tratamiento estandarizado en todos los niños con esofagitis o gastropatía erosiva y dispepsia funcional, lo que puede sesgar el resultado del seguimiento, sin embargo, pone sobre la mesa de discusión el papel que juega el SBID en el desarrollo de la dispepsia en niños.

\section{Conclusión}

La dispepsia en niños sigue siendo un tema que requiere más investigación, sin embargo, de acuerdo con lo encontrado en nuestro estudio consideramos que se deben descartar primero causas orgánicas antes de catalogar al niño con dispepsia funcional. En términos generales consideramos que es necesario un abordaje escalonado que incluya endoscopia con toma de biopsia, búsqueda de $H$. pylori y una prueba de hidrogeniones

\section{Financiamiento}

Este estudio no recibió financiamiento.

\section{Conflicto de intereses}

Los autores de este estudio declaran no tener conflicto de intereses.

\section{Responsabilidades éticas}

Protección de personas y animales. Los autores declaran que los procedimientos seguidos se conformaron a las normas éticas del comité de experimentación humana responsable y de acuerdo con la Asociación Médica Mundial y la Declaración de Helsinki.

Confidencialidad de los datos. Los autores declaran que han seguido los protocolos de su centro de trabajo sobre la publicación de datos de pacientes.

Derecho a la privacidad y consentimiento informado. Los autores han obtenido el consentimiento informado de los pacientes y/o sujetos referidos en el artículo. Este documento obra en poder del autor de correspondencia.

\section{Bibliografía}

1. Tack J, Talley NJ. Functional dyspepsia-symptoms, definitions and validity of the Rome III criteria. Nat Rev Gastroenterol Hepatol. 2013;10:134-41.

2. Tack J, Talley NJ, Camilleri M, Holtmann G, Hu P, Malagelada JR, et al. Functional gastroduodenal disorders. Gastroenterology. 2006;130:1466-79.

3. Mani J, Madani S, Thomas R. Economic impact and prognostic factors of functional dyspepsia in children. J Pediatr Gastroenterol Nutr. 2020;70(4):e65ロe70.

4. Lewis ML, Palsson OS, Whitehead WE, van Tilburg MAL. Prevalence of functional gastrointestinal disorders in children and adolescents. J Pediatr. 2016:177:39п43.e3.

5. Lacy BE, Weiser KT, Kennedy AT, Crowell MD, Talley NJ. Functional dyspepsia: the economic impact to patients. Aliment Pharmacol Ther. 2013;38(2):17007.

6. Rasquin A, Di Lorenzo C, Forbes D, Guiraldes E, Hyams JS, Staiano A, et al. Childhood functional gastrointestinal disorders: child/adolescent. Gastroenterology. 2006:130(5):1527ロ37.

7. Kleinman R, Goulet O, Mieli-Vergani G, Sanderson I, Sherman P, Shneider B, editores. Walker's Pediatric Gastrointestinal Disease.Vol. 1. People's Medical Publishing House; 2018. pp. 153-161.

8. Ocampo del Prado L, Cervantes Bustamante R, Ramírez Mayans JA Dolor abdominal crónico recurrente en niños. Acta Pediatr Mex. 2003;24(6):351-3

9. Méndez Nieto CM, Ramírez-Mayans J, Cervantes R, Mata N, Cuevas F, Martínez C, et al. Diagnóstico de Helicobacter pylori en niños con dolor abdominal recurrente. Acta Gastroent Latinoamer. 1994;24:169-74.

10. Jones NL, Koletzko S, Goodman K, Bontems P, Cadranel S, Casswall T, et al. Joint ESPGHAN/NASPGHAN Guidelines for the Management of Helicobacter pylori in Children and Adolescents (Update 2016). J Pediatr Gastroenterol Nutr. 2017;64(6):99101003.

11. Hyams JS, Davis P, Sylvester FA, Zeiter DK, Justinich CJ, Lerer T. Dyspepsia in children and adolescents: a prospective study. J Pediatr Gastroenterol Nutr. 2000;30(4):413ロ8.

12. Adeniyi OF Odeghe EA, Lawal MA, Olowu AO, Ademuyiwa A. Recurrent abdominal pain and upper gastrointestinal endoscopy findings in children and adolescents presenting at the Lagos University Teaching Hospital. PLoS One. 2019;14(5):e0216394.

13. Spee LA, Madderom MB, Pijpers M, van Leeuwen Y, Berger MY. Association between Helicobacter pylori and gastrointestinal symptoms in children. Pediatrics. 2010;125(3):e651ロe669. 
14. Farrell S, Milliken I, Murphy JL, Wootton SA, McCallion WA. Non ulcer dyspepsia and Helicobacter pylori eradication in children. J Pediatr Surg. 2005;40(10):1547ロ50.

15. Ünlüsoy Aksu A, Yılmaz G, Eğritaș Gürkan Ö, Sarı S, Dalgıç B. The effect of Helicobacter pylori eradication on functional dyspepsia in Turkish children. Helicobacter. 2018;23(4):e12497.

16. Petzold G, Amanzada A, Gress TM, Ellenrieder V, Neesse A, Kunsch S High prevalence of pathological hydrogen breath tests in patients with functional dyspepsia. Digestion. 2019;100(3):186-91.

17. Shimura S, Ishimura N, Mikami H, Okimoto E, Uno G, Tamagawa $Y$, et al. Small intestinal bacterial overgrowth in patients with refractory functional gastrointestinal disorders. J Neurogastroenterol Motil. 2016;22(1):60-8.
18. Avelar Rodriguez D, Ryan PM, Toro Monjaraz EM, Ramirez Mayans JA, Quigley EM. Small intestinal bacterial overgrowth in children: A state-ofthe-art review. Front Pediatr. 2019;7:363.

19. Shah A, Talley NJ, Koloski N, Macdonald GA, Kendall BJ, Shanahan ER, et al. Duodenal bacterial load as determined by quantitative polymerase chain reaction in asymptomatic controls, functional gastrointestinal disorders and inflammatory bowel disease. Aliment Pharmacol Ther. 2020; 52(1):155-67.

20. Tziatzios G, Giamarellos-Bourboulis EJ, Papanikolaou IS, Pimentel M, Dimitriadis GD, Triantafyllou K. Is small intestinal bacterial overgrowth involved in the pathogenesis of functional dyspepsia? Med Hypotheses. 2017; 106:26-32. 